\title{
Multiple family group treatment reduces distress for caregivers of people with schizophrenia
}

Hazel NA, McDonell MG, Short RA, et al. Impact of multiple-family groups for outpatients with schizophrenia on caregivers' distress and resources. Psychiatr Serv 2004;55:35-41.

Does multiple family group treatment reduce stress and increase psychosocial resources in family caregivers of people with schizophrenia?

\section{METHODS}

-

Design: Randomised controlled trial

Allocation: Concealed

Blinding: Nature of intervention precludes blinding of participants; blinding of assessors not stated.

$\sum\langle$

Follow up period: Two years.

Setting: A large community mental health centre in Washington State, USA; 1996 to 2001.

Patients: Ninety seven people living in the community (mean age 32 years) with schizophrenia (64), schizoaffective disorder (32) or other psychotic disorders (1) according to DSM-IV criteric. Their corresponding family-member caregivers were mothers (69), siblings (12), spouses/partners (7), children (3), other (4; data missing for two participants).

R

Intervention: Participants were randomised to multiple family group treatment (53) or usual care (44), and organised into seven cohorts. Multiple family group treatment involved two clinicians and six to eight families integrating psychoeducation and behavioural family therapy in 90 minute group format sessions twice a month (year 1) or monthly (year 2). Year 1 focussed on relapse prevention and year 2 on social and vocational rehabilitation. All participants received standard care at an outpatient mental health centre.

Outcomes: Caregivers' distress: using the Perceived Stress Scale (PSS), Anger Expression Scale (Ax), Global Distress Index of the Center for Epidemiologic Studies Depression Scale (CES-D), and 20 items from the State-Trait Anxiety Inventory (SAI). Caregivers' resources: using the Interpersonal Support Evaluation List (ISEL), Social Support Questionnaire (SSQ), and Revised Ways of Coping Checklist (RWCCL).

Patient follow up: $78 \%$ (21 participants dropped out (12 intervention, nine control).

\section{MAIN RESULTS}

Multiple family group treatment significantly reduced caregiver distress compared with usual care (mean difference scores between distress at baseline and year 2, multiple family group treatment $v$ usual care: $-0.06 v 0.09$ ). There were no significant differences in caregivers resources between groups at 2 years. Mental Illness: Research and Training, Washington State University, Spokane, Washington, USA; dyck@wsu.edu

Sources of funding: the National Institute of Mental Health.

\section{CONCLUSIONS}

Although multiple family group treatment reduced caregiver distress, it did not increase caregivers resources compared with usual care.

\section{NOTES}

Caregivers in the multiple family group treatment had significantly lower levels of baseline distress than caregivers in the usual care group $(\mathrm{p}<0.05)$. Although these baseline differences were controlled for using repeated measures analyses of covariance on the principle outcome variables, this may affect generalisability of the findings.

\section{Commentory}

$\mathrm{n}$ the mid 1950s, the theory of expressed emotions (EE) was developed in an attempt to describe caregiver attitude. ${ }^{1}$ The EE concept has proven useful for the understanding of the interactions within families with a member suffering from schizophrenia. It has also resulted in various therapeutic and educational strategies aiming at reducing symptom intensities of the psychotic patients, as well as reducing relapse rates.

In later years, EE related research has also focused on the burden of the whole family, not only of the patients themselves. Quite a number of randomised trials have indicated that family based interventions (patient included) promote family wellbeing, in addition to lowered relapse rates and improved recovery of patients. ${ }^{2}$ Such interventions include elements of stress management, communication strategies, problem solving, goal achievement, and psychoeducation about the psychotic disorder and early warning signs.

The implementation of family intervention techniques in routine practice has been hindered, however, for several reasons - one important reason being staff limitations. Applying psychoeducation on multiple family groups ${ }^{4}$ is, therefore, a welcome contribution in the field of family intervention. Dr Dennis Dyck and his research group at Washington State University have put considerable effort into validating the technique. Their recent, careful study provides excellent evidence for the valid use of multiple family group treatment in reducing caregiver distress in families with psychotic patients.

Some interesting perspectives emerge. Although the usefulness of single family based intervention techniques is well established, multiple family group treatment may be of particular interest within the limits of restricted budgets. Also, the possible applicability of the technique should be evaluated further with regard to other chronic disorders, especially highly prevalent disorders, such as Alzheimer's disease ${ }^{5}$ and vascular dementia.

Åke Edman, MD, PhD

Göteborg University, Institute of Clinical Neuroscience, Psychiatry Section, Mölndal, Sweden

1 Brown GW, Carstairs GM, Topping G. Post-hospital adjustment of chronic mental patients. Lancet 1958:2:685-8.

2 McFarlane WR, Dixon L, Lukens E, et al. Family psychoeducation and schizophrenia: a review of the literature. J Marital Fam Ther 2003;29:223-45.

3 Falloon IR, Boyd JL, McGill C. Family care of schizophrenia. New York: The Guilford Press, 1984

4 McFarlane WR. Multiple family groups in the treatment of severe psychiatric disorders. New York: The Guilford Press, 2002.

5 Marriott A, Donaldson C, Tarrier N, et al. Effectiveness of cognitive-

behavioural family intervention in reducing the burden of care in carers of patients with Alzheimer's disease. Br J Psychiatry 2000;176:557-62. 